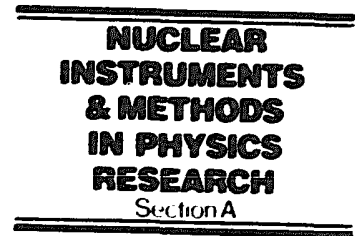

\title{
Space charge effects in circular electron beams
}

\author{
Eltjo H. Haselhoff and Gerard J. Ernst \\ Nederlands Centrum ioor Laser Research, Postbus 2662, 7500 CR Enschede. The Netherlands
}

In this paper we discuss the effects of the space charge fields of centripetally accelerated particles. It is shown that these effects may cause a discrepancy in terms of the calculated rms emittance as compared to calculations where space charge fields corresponding to a uniform motion of the electrons are used.

\section{Introduction}

It is well known that at relativistic velocities the electric field of a point charge concentrates along the directions perpendicular to its motion [1]. When the charge moves at a constant velocity, the space charge field is symmetric with respect to the plane normal to the velocity vector. Since in most electron accelerators (linacs) the electron velocity is more or less uniform during the transit time, this "uniform field" is a convenient approximation for space charge calculations. In the case of a centripetal acceleration however, like in a bending magnet, the symmetry of the fields disappears, and both the strength and the shape of the space charge field will depend not only on the energy, but also on the point of observation and the bending radius. In this paper we investigate the discrepancy that arises with respect to the beam emittance evolution due to this "deformation" of the space charge fields in a circular bend.

An application of the theory concerns the design of a $6 \mathrm{MeV}$ electron beam spectrometer for the TEUFEL (Twente/Eindhoven university and UCN Free Electron Laser) project.

\section{The space charge fields of moving charges}

In this section we briefly repeat the space charge theory as given in many text books (c.g. ref. [2]). Consider a point charge $q$ moving in a straight line with constant velocity $l(t)$. Its space charge field amounts to (SI units):

$E(\xi, t)=\frac{q}{4 \pi \epsilon_{0}} \frac{r}{r^{* 3}}\left(1-r^{2} / c^{2}\right)$,

where $\xi$ denotes the position of the observer at the time of observation $t, r$ is a vector pointing from the particle's position at time $t$ to the position of the observer, $r$ is the particle's velocity and

$r^{*}=r^{\prime}-\frac{\boldsymbol{r}^{\prime} \cdot \boldsymbol{r}^{\prime}}{c}$,

in which the prime implies retarded values and $c$ is the velocity of light. The magnetic field component due to the particle's motion can be written as

$\boldsymbol{B}(\xi . t)=\frac{1}{c^{2}} \boldsymbol{l}^{\prime} \times \boldsymbol{E}$.

As for convenience, we define a normalised electric field

$\mathscr{E} \equiv 4 \pi \epsilon_{0} r^{2}|E| / q$,

which cancels the $1 / r^{2}$-dependence. This field amounts to the actual electric field, relative to the field that would be observed if the particle were standing still. As an illustration, fig. 1 shows the normalised electric field $f$ of a uniformly moving charge, as a function of the observation angle $\varphi$, for different energies $\gamma$ (units of rest mass energy). Fig. 2 shows the position and motion

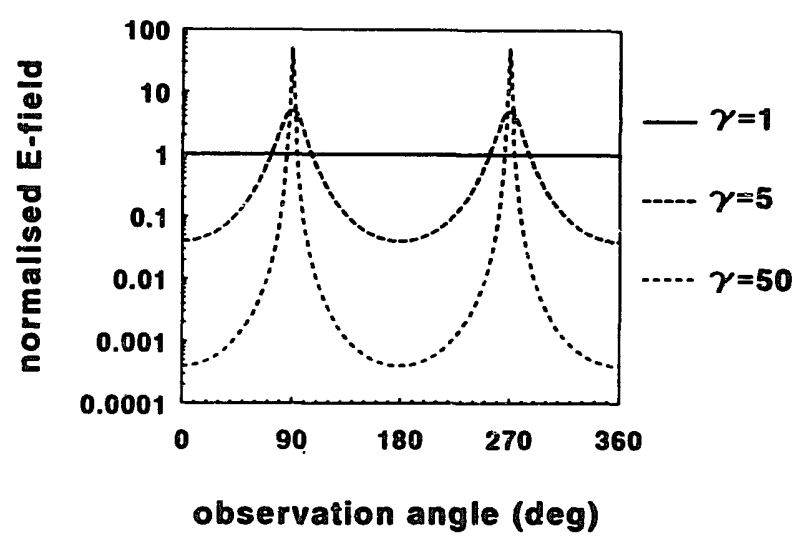

Fig. 1. Normalised electric field $\left(E\left(4 \pi \epsilon_{11} r^{2} / q\right)\right)$ of a charged particle with uniform velocity. for three different energies (units of $m_{11} c^{2}$ ), as a function of the ohservation angle $\varphi$ (see fig. 2). 


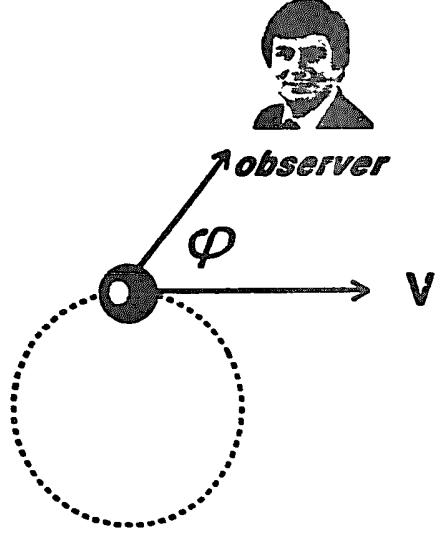

Fig. 2. Circular motion of the point charge, and position of the observation point under the observation angle $\varphi$.

of the charge and the point of observation. When the particle is standing still, the electric field has a circular ( = spherical) shape, while for high energies the fields concentrate perpendicularly to the particle's motion.

The electromagnetic field as given by eqs. (1) and (2) is only exact when the particie moves with a uniform velocity. For an arbitrary motion, the fields take a more complicated form:

$$
\begin{aligned}
E(\xi, t)= & \frac{q}{4 \pi \epsilon_{0}} \frac{1}{r^{* 3}}\left\{\left(r^{\prime}-\frac{r^{\prime} v^{\prime}}{c}\right)\left(1-\frac{r^{\prime 2}}{c^{2}}\right)\right. \\
& \left.+\frac{1}{c^{2}} r^{\prime} \times\left[\left(r^{\prime}-\frac{r^{\prime} v^{\prime}}{c}\right) \times i^{\prime}\right]\right\}, \\
B(\xi, t)= & \frac{r^{\prime} \times E}{r^{\prime} c} .
\end{aligned}
$$

In many electron space charge calculations, the expressions (1) and (2) are sufficient, because of the rapid acceleration of electrons close to the yelocity of light, and the near-parallel motion, like e.g. in linear accelerators. Also in circular accelerators and beam transport devices like bending magnets, a common approach is to take the instantaneous values of the particles' positions and velocities, using the expressions (1) and (2), and add the external field that cause the bend, the way it is done in e.g. the well-known particle code PARMELA.

A more accurate approach however, is to use the full expressions (3) and (4). We will now consider this complete space charge field of a point charge, assuming a perfect circular motion, as caused by c.g. a uniform magnetic field. Although the case of a real electron beam never results in a perfect circular motion (due to the space charge effects themselves), this is still a convenient approximation.

We define an orthogonal coordinate system $\left(e_{1}, e_{1}, e_{i}\right)$ and consicier a point charge performing the circular motion with radius $R$, given by

$$
\begin{aligned}
& x(t)=R \sin \left(\frac{t}{R} t\right), \\
& y(t)=R \cos \left(\frac{t}{R} t\right), \\
& z(t)=\text { constant. }
\end{aligned}
$$

The main problem in determining the expressions (3) and (4), is to find the particle's retarded position for an observer in $\left(\xi_{a}, \xi_{1}, \xi_{z}\right)$ at time $t$, i.c. to find the time $t^{\prime}$ so that

$$
\begin{aligned}
& \left(x\left(t^{\prime}\right)-\xi_{x}\right)^{2}+\left(y\left(t^{\prime}\right)-\xi_{y}\right)^{2}+\left(z\left(t^{\prime}\right)+\xi_{z}\right)^{2} \\
& \quad=c^{2}\left(t-t^{\prime}\right)^{2} .
\end{aligned}
$$

When the particles are distributed in a bunch with finite dimensions, an extra complication arises, because of the different rotation centers of each particle. This problem can be cancelled by considering the displacement of each particle during the time interval $\Delta t \equiv t-$ $t^{\prime}$, which is independent of its rotation center, and which is given by

$$
\begin{aligned}
& \Delta x=R\left(-\sin \left(\frac{l}{R} \Delta t\right) \frac{l_{x}}{l}+\left(1-\cos \left(\frac{l^{\prime}}{R} \Delta t\right)\right) \frac{l_{y}}{l^{v}}\right) \text {, } \\
& \Delta y=R\left(-\sin \left(\frac{v}{R} \Delta t\right) \frac{v_{y}}{l}-\left(1-\cos \left(\frac{l}{R} \Delta t\right)\right) \frac{l_{A}}{l}\right) \text {, } \\
& \Delta z=-r \cdot \Delta t \text {. }
\end{aligned}
$$

Given the coordinates of the particle at time $t, x(t)$, $y(t)$ and $z(t)$, the retarded time for the observation point $\left(\xi_{x}, \xi_{v}, \xi_{i}\right)$ is then defined by

$$
\left|\left(\begin{array}{l}
x(t)+\Delta x \\
y(t)+\Delta y \\
z(t)+\Delta z
\end{array}\right)-\left(\begin{array}{l}
\xi_{x} \\
\xi_{y} \\
\xi_{z}
\end{array}\right)\right|=c \Delta t .
$$

An analytic expression for the system (5) has not yet been derived, but a numerical Newton algorithm [3] has been found to be quite effective.

Because the shape of the fields (3) and (4) only depends on the retarded position of the point charge, it can easily be seen from a geometrical point of view that the azimuthal dependence of the normalized electric field $\mathscr{E}$ as defined above, is identical for constant ratios of the bending radius $R$ and the distance $r$ between the observer and the particle at the time of observation. This suggests the definition of the dimensionless parameter

$s=r / R$.

Fig. 3 shows the azimuthal dependence of $\&$ for a charge with energy $\gamma=5$, in the case of a circular motion as descrioud above. At short observation distances and/or large bending radii $\left(s=10^{-4}\right)$ the solution tends to the previous case. shown by the middle 


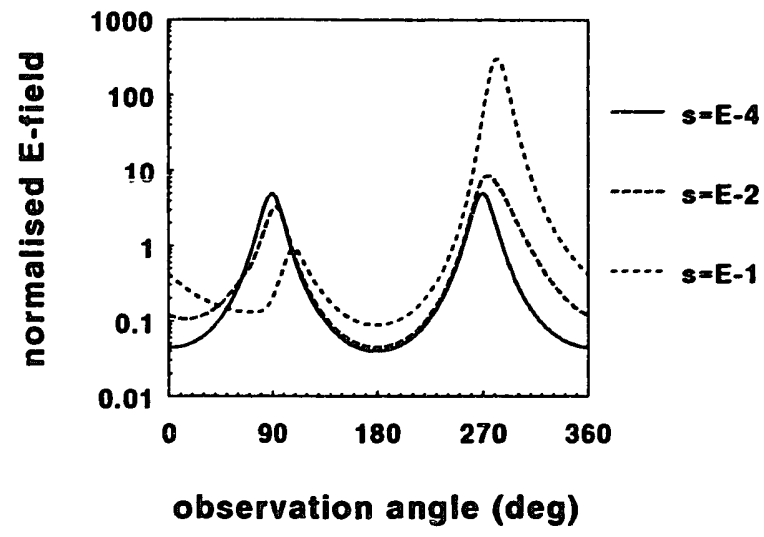

Fig. 3. Normalised electric field of a charged particle performing a circular motion. as a function of the observation angle, for three different ratios of the observation distance $r$ and the radius of curvature $R(s \equiv r / R)$. The energy $\gamma=5$.

curve in fig. 1. At longer distances and/or small bending radii however an asymmetry arises, as shown by the second and third curve in fig. 3. Note that the electric field is stronger at the "inside" of the circular motion, while the maximum value tends to shift from $270^{\circ}$ (perpendicular to the particle's motion) to the right. At relatively large observation distances it can be shown that this maximum shifts all the way to $360^{\circ}$ (in the direction of the particle's motion). In this case, only the second term in eq. (3) effectively contributes to the fields, since it falls off with $1 / r$ rather than $1 / r^{2}$, like the first term. (In the fact, the second term denotes the synchrotron radiation emitted from the particle.)

The energy dependence of the field disturbation is shown in fig. 4 , for $s=0.01$. For a particle at rest the electric field is spherical; for $\gamma=5$ we find the same solution as shown in fig. 3 , while for higher energies the asymmetry is found to get considerably stronger.

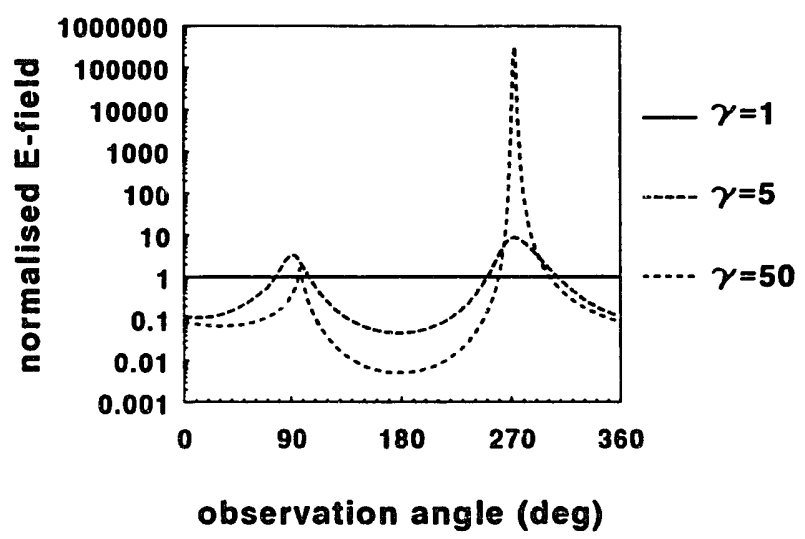

Fig. 4. Normalised electric field of a charged particle performing a circular motion, as a function of the observation angle. for three different energies $\gamma$ (units of $m_{10} c^{2}$ ). for $s=1 \times 10^{-2}$

\section{The particle tracing code}

In the case of many interacting particles, like an electron pulse propagating through a bending magnet, the net effect of the phenomena described in the previous section is hard to predict. At short distances the space charge field will tend to the "uniform case" as given by eqs. (1) and (2), while the interaction is much stronger than the interaction with more distant particles, because of the $1 / r^{2}$-dependence. This fact could be used to justify the use of eqs. (1) and (2) rather than the full expressions. On the other hand, the number of distant particles, with asymmetric space charge fields, is larger (depending also on the pulse dimensions). For high energies, the asymmetry is found to get quite strong, while simultaneously the overall effect of space charge will reduce, so that the question remains: does this high-energy asymmetry in the fields still have considerable effect?

In order to answer these questions, we created a particle tracing code, similar to the PAPA program as reported elsewhere [4], which includes the complete field expressions (3) and (4). The program solves for the positions $k_{i} \equiv\left(x_{i}, y_{i}, z_{i}\right)$ and momenta $P_{i} \equiv$ $\left(P_{x}, P_{y}, P_{z}\right)=\gamma\left(v_{x}, v_{y}, v_{z}\right)$ of $\mathscr{r}$ "macro-electrons" propagating in a homogeneous magnetic field:

$\dot{\boldsymbol{k}}_{i}=\frac{\boldsymbol{P}_{i}}{\gamma_{i}}$

$\dot{\boldsymbol{P}}_{i}=\frac{-\boldsymbol{e}}{m_{0}}\left(\boldsymbol{E}+\frac{\boldsymbol{P}_{i}}{\gamma_{i}} \times \boldsymbol{B}\right)$,

with $i=1 \ldots . t$, where the dot denotes the total time derivative, $\gamma_{i}=\left(\left|P_{1}\right|^{2} / c^{2}+1\right)^{1 / 2}, m_{0}$ and $e$ are the rest mass and charge of an electron respectively, and $E$ and $B$ are the sum of all space charge contributions of the other particles with index $j=1 \ldots ., j \neq i$, derived as explained above, plus the homogeneous magnetic field. All the results presented here will be in terms of the one-dimensional rms emittance, commonly defined as

$4 \pi\left(\left\langle x^{2}\right\rangle\left\langle x^{\prime 2}\right\rangle-\left\langle x x^{\prime}\right\rangle^{2}\right)^{1 / 2}$

where $x$ denotes the distance from the mass center of the pulse, and $x^{\prime}$ the angle between the particle's velocity and the average velocity.

\section{4. "Inherent" emittance growth in a magnetic bend}

When a "perfect" (zero emittance) electron beam is bent through a homogeneous magnetic field, the rms emittance will always increase, even if there are no space charge interactions at all. When the central orbit of the bend has a radius $R$. and an electron enters the 

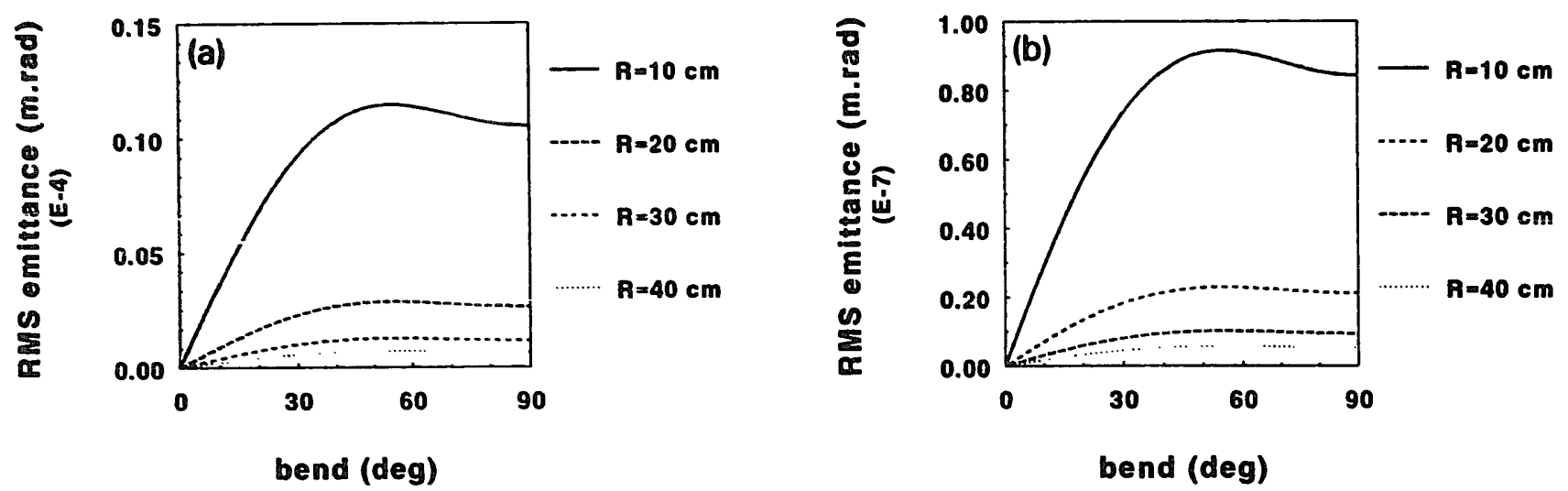

Fig. 5. "Inherent" emittance growth (no space charge interactions) in a circular bend, as a function of the bending angle, for different hending radii. (a) Beam radius: $5 \mathrm{~mm}$. (b) Beam radius: $1 \mathrm{~mm}$.

bending magnet perpendicularly at a distance $d_{0}$ from the central orbit, some simple algebra reveals that the distance between the electron and the central orbit after a bend $\varphi$ is given by

$d=R-\sqrt{R^{2}-d_{0}^{2} \sin ^{2} \varphi}+d_{0} \cos \varphi$,

while the tangent of the angle $\vartheta$ between the electron's velocity and the central orbit amounts to:

$\tan (\vartheta)=\frac{d_{0} \sin \varphi}{\sqrt{R^{2}-d_{0}^{2} \sin ^{2} \varphi}}$.

Because there is no linear dependence between $d$ and $\tan (\vartheta)$, the rms emittance as defined by eq. (6) will always increase in the plane of rotation, apparently at a faster rate for large values of $d_{0} / R$. This is shown numerically in fig. 5a, which shows the rms emittance evolution of an electron pulse being bent in a homogeneous magnetic field. Initially the pulse had zero emittance, and a radius of $5 \mathrm{~mm}$. There were no space charge interactions at all, the only force acting on the electrons arose from the magnetic field. Indeed it is found that larger values of the bending radius $R$ decreases the "inherent" emittance growth. Fig. 5b shows the same calculation for a smaller beam radius $(1 \mathrm{~mm})$. The inherent emittance growth is now smaller than most "common" beam emittances. Comparison of both figures explains why many beam transport designs focus the electron beam at the entrance edge of bending magnets: not because of any space charge effect, but as to reduce the inherent emittance growth.

\section{Numerical results}

In this section we will compare three different cases: (i) the emittance growth of an initially "perfect" electron beam during free propagation in a straight line. having an initial velocity distribution

$r_{z}(t=0)=r_{z: 0}$,

$r_{x}(t=0)=-r_{z} 2 x /(\pi R)$,

$r_{y}(t=0)=0$,

so that the beam will reach a focus in the $x$-direction after a drift length of $\pi R / 2$, (ii) the emittance growth of a perfect electron beam being bent in a homogeneous magnetic field using the linear space charge eqs. (1) and (2) (this pulse will also focus in the radial direction after a bend of $\pi / 2$ corresponding to a drift length of $\pi R / 2$, identical to the previous case), and (iii) the same as (ii), but now using the complete space charge expressions as described before. In all calculations we used a beam radius of $1 \mathrm{~mm}$, which sufficiently cancels the "inherent" emittance growth as discussed earlier.

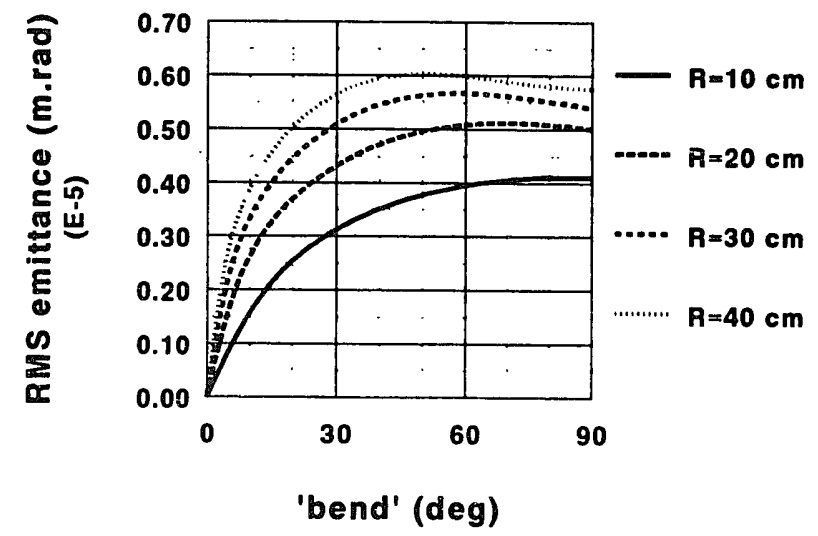

Fig. 6. Rms emittance growth of a uniformly moving electron pulse, as a function of the drift distance. The drift distance is specified in terms of the angle that would correspond to the drift during a circular bend with radius $R$. The initial velocilies were chosen as to obtain a focus after a drift of " $90^{\circ}$ ". heam radius $=1 \mathrm{~mm}, \gamma=12$. 

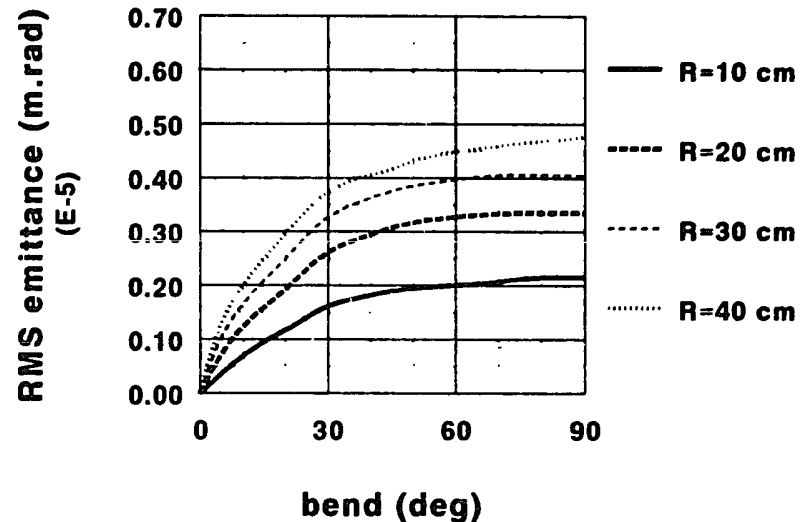

Fig. 7. Rms emittance growth during a $90^{\circ}$ bend, using "linear" space charge fields, for differet.t bending radii. Beam radius $=1 \mathrm{~mm}, \gamma=12$.

Fig. 6 shows the emittance growth in the case of linear drift, for four different drift/focus distances, denoted by the "bending radii" which would correspond to the case of a circular motion. The beam current was $1 \mathrm{~A}$, at an energy of $y=12$. Note that halfway the drift with $R=40 \mathrm{~cm}$ (i.e. after a drift of $40(\pi / 4) \mathrm{cm})$ the emittance is not necessarily equal to the value at the end of the $R=20 \mathrm{~cm}$ case (corresponding to he same drift length). This is because the four cases have no identical evolution, since the initial velocity distributions are different as to obtain the different focus distances.

Fig. 7 shows the emittance evolution when the same beam is bent by a homogeneous magnetic field, still assuming the same space charge interaction as in the previous case. The bending radii were 10, 20, 30 and 40 $\mathrm{cm}$. It is found that the rms emittance now grows a little slower, especially for small bending radii. Apparently, continuously "guiding" the electrons into a focus as was done here, maintains the beam quality better than in the previous case, where the electrons were given only an initial "push" towards a focus and then drift freely.

Fig. 8 shows the results for the same bend, but with the use of the full field expressions (3) and (4). Comparison with fig. 7 reveals the interesting fact that now there is no longer a proportional relation between the bending radius and the emittance growth. For large bending radii the emittance evolution seems to tend to the previous case, as expected, but for small radii of curvature the "acceleration terms" in eq. (3) seem to cancel the positive effect of a shorter drift distance. The optimal bending radius in this case, taking the maximum profit of the dilemma of short drift distance versus circular space charge effects. is found to be around $20 \mathrm{~cm}$. As to get a more explicit ide: of the

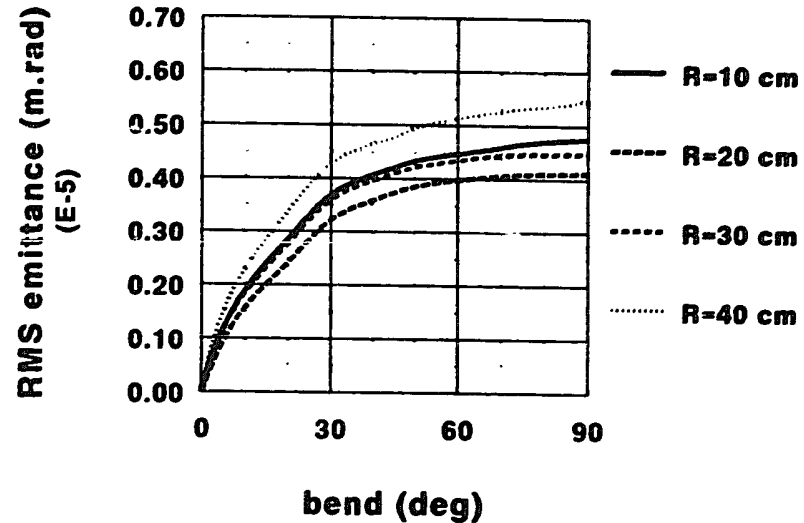

Fig. 8. Rms emittance growth during a $900^{\circ}$ bend. using the exact space charge expressions (3) and (4), for different bending radii. Beam radius $1 \mathrm{~mm}, \gamma=12$.

influence of the bending radius, we performed a number of simulations as described before, and plotted the rms emittance after a bend of $80^{\circ}$ as a function of the corresponding bending radius $R$. The results are shown in fig. 9, showing clearly a minimum in the emittance curve, at a radius of approximately $20 \mathrm{~cm}$.

The optimal radius as found here is probably quite specific. We found no dependence on the beam current, but there is a dependence on the energy and especially on the electron pulse's dimensions. The various degrees of freedom make a parametric study difficult, especially because the full space charge calculations are very time consuming. A similar optimisation study as described above was done for the case of a spectrometer magnet, to be used as a diagnostic tool for our $6 \mathrm{MeV}$ FEL injector linac. Assuming pulses of $5 \mathrm{~mm}$ radius and $20 \mathrm{ps}$ length, we found an optimal bending radius of approximately $25 \mathrm{~cm}$.
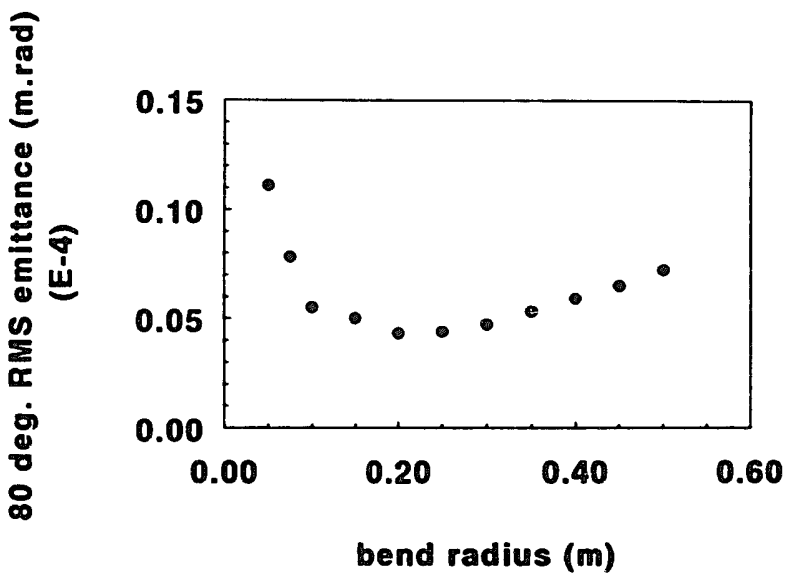

Fig. 9. Rms emittance after a $80^{\circ}$ bend, as a function of the bending radius, using the exact space charge expressions (3) and (4). Beam radius $1 \mathrm{~mm} . \gamma=12$. 


\section{Conclusions}

We observed that the space charge fields of uniformly moving charges are not always sufficient to describe the behaviour of charged particles during centripetal acceleration. Although at short distances, where the space charge interactions are the strongest, the fields corresponding to uniform and accelerated motion are more or less equal, the macroscopic behaviour of a pulse of macro-electrons can still be different.

There seems to exist an "optimal" value for the bending radius, optimising for the dilemma of large bending radii implementing a long drift distance, and small radii implying strong space change forces, both ruining the beam quality. This optimal value for the bending radius also depends on the pulse dimensions and the energy and should be determined for each individual case.

\section{References}

[1] E.g. J.R. Reitz, Foundations of Electromagnetic Theory (Addison-Wesley, 1979) chap. 21.

[2] J.D. Jackson, Classical Electrodynamics (Wiley, New York. 1962) chap. 14.

[3] W.H. Press et al., Numerical Recipes (Cambridge University Press, 1988).

[4] E.H. Haselhoff and G.J. Ernst, Nucl. Instr. and Meth. $\therefore 296$ (1990) 744. 